\title{
Los genes TAP y LMP2 en el asma alérgica
}

\author{
Beatriz Martínez, Luis Caraballo
}

\begin{abstract}
Resumen
Los pacientes con asma alérgica presentan una fuerte respuesta de IgE contra los alergenos de ácaros; por tanto, estudiar la inmunogenética de esta respuesta podría ayudar a entender la etiología de las enfermedades alérgicas. El asma alérgica es una enfermedad muy común en el trópico, siendo los principales agentes causales los ácaros Blomia tropicalis y Dermatophagoides pteronyssinus. En la población mulata de Cartagena, hemos descrito previamente asociaciones entre los genes HLA clase II y la respuesta de IgE a los alergenos de ácaros en pacientes asmáticos.
\end{abstract}

Los genes TAP y LMP intervienen en el procesamiento antigénico pero su posible papel en la patogénesis de asma alérgica no está bien documentada. En este estudio, evaluamos el efecto del polimorfismo de los genes TAP y LMP en el control de la respuesta de IgE específica contra alergenos de ácaros en dos grupos: 96 pacientes asmáticos no relacionados y 97 controles no alérgicos, pertenecientes al grupo étnico mulatos. La tipificación de TAP1 y TAP2 fue hecha por PCR/SSO y del gen LMP2 por PCR/RFLP. La frecuencia de cada alelo en pacientes con asma alérgica y controles fue: TAP $^{*} 0101: 52,6-56,2 \%$; TAP ${ }^{*} 02011:$ 35,9-34,9\%; TAP*0301: 5,2-3,8\%; TAP*0401: 6,3-4,7\%; TAP2A: 31,5-30,5\%; TAP2B: 44,3-46,6\%; TAP2C: 16,5-18,0\%; TAP 2H: 7,7-4,9\%; LMP2*H/H: 6,7-10,4\%; LMP2*R/R: 42,7-44,8\%; LMP2*R/H: 50,6-44,8\%.

No hubo ninguna diferencia estadísticamente significativa en la distribución de estos alelos entre los dos grupos estudiados. Además, no hallamos desequilibrio de ligamiento entre estos genes y los alelos HLA DRB1. Estos hallazgos sugieren que los genes TAP y LMP2 no están comprometidos primariamente con la susceptibilidad genética del asma alérrgica.

\section{Summary}

Patients with mite-induced allergic asthma (AA) present strong IgE-response to mite allergens. Thus, immunogenetic study of this response could help in the understanding of allergic disease etiology. AA is a common disease in the Tropics, Blomia tropicalis and Dermatophagoides pteronyssinus being the principal causative agents. An association between HLA class II genes and IgE-response to mite allergens in AA patients from the prevalent ethnic mulatto group in Cartagena, a Caribbean city, has already been described (by these authors), the main vector being Blomia tropicalis. TAP and LMP genes play an important part in antigen processing but their possible role in AA pathogenesis has not been well-documented. In this study, the role of TAP and LMP2 polymorphism in the control of specific lgE-response to mite allergens has been evaluated in two groups, consisting of 96 unrelated asthmatic patients with IgE-response to mite allergens and 97 non-allergic controls belonging to the mulatto ethnic group. TAP1 and TAP2 were typed by PCR/SSO; LMP2 was typed by PCR/RFLP. Each alleles' frequency in AA patients and controls was:

Instituto de Investigaciones Inmunológicas, Universidad de Cartagena, Cartagena, Colombia. 
TAP*0101: 52.6-56.2\%; TAP*02011: 35.9-34.9\%; TAP*0301: 5.2-3.8\%; TAP ${ }^{*} 0401$ : 6.3-4.7\%; TAP2A: 31.5-30.5\%; TAP2B: 44.3-46.6\%; TAP2C: $16.5-18.0 \%$; TAP 2H: 7.7-4.9\%; LMP2*H/ H: 6.7-10.4\%; LMP2*RR: 42.7-44.8\%; LMP2*RH: 50.6-44.8\%. There was no significant statistical difference in allele distribution between the two groups studied. In addition, no linkage disequilibrium between the HLA DRB1 allele and TAP and LMP2 genes was found. These findings suggest that TAP and LMP genes are not primarily involved in AA genetic susceptibility.

Recientemente se han identificado nuevos genes en la región clase II del sistema HLA. Entre ellos, dos genes inducibles por el gamma-interferón, denominados TAP1 y TAP2 (transporter associated with antigen), que codifican proteínas que participan en el procesamiento antigénico (14) y dos genes que codifican dos subunidades del proteasoma, los cuales han sido denominados LMP2 y LMP7 (low molecular weight polypeptides). El análisis de su polimorfismo ha demostrado que estos genes están relacionados con la susceptibilidad a padecer algunas enfermedades de tipo inmunológico (5-8).

Los pacientes con asma alérgica inducida por ácaros tienen una fuerte respuesta de $\operatorname{lgE}$ a alergenos de ácaros, lo cual parece ser crucial para la inducción y mantenimiento de la inflamación bronquial que causa los síntomas de la enfermedad. Por lo anterior, el estudio de la inmunogenética de esta respuesta podría ayudar a entender la etiología del asma y otras enfermedades alérgicas. El asma alérgica es una enfermedad común. En el trópico, donde el grupo étnico sobresaliente es mulato (9), el principal sensibilizador es el ácaro Blomia tropicalis. En esta población, nosotros hemos descrito previamente asociaciones entre los genes clase II del sistema HLA y la respuesta IgE contra alergenos de ácaros en pacientes asmáticos (10-12).

Este estudio fue hecho para analizar la asociación entre la respuesta de IgE contra alergenos de ácaros y los alelos TAP y LMP2, describir la distribución de estos alelos y determinar si hay desequilibrio de ligamiento con respecto a los genes HLA DRB1 y HLA DQB1 en una población colombiana mulata normal y en pacientes con asma alérgica, ya que se han descrito desequilibrios de ligamientos entre los genes TAP, LMP y los alelos HLA $(8,13)$. Analizamos, además, si dicho fenómeno estaba presente es-pecíficamente con aquellos alelos HLA previamente asociados con la respuesta de IgE a alergenos de ácaros, tales como HLA DRB ${ }^{\star} 03$ y HLA DQB1*0601 (12).

\section{Materiales y métodos}

Pacientes y controles: los grupos de estudio fueron 96 pacientes asmáticos no relacionados, con respuesta de IgE contra alergenos de ácaros y 97 controles no alérgicos, ambos pertenecientes al grupo étnico mulato. El asma alérgica fue diagnosticada siguiendo los criterios sugeridos por un panel de expertos de la Fundación Americana de Alergia (14). Todos los pacientes tuvieron síntomas relacionados con la exposición al polvo casero y eran altamente alérgicos a $B$. tropicalis y $D$. pteronyssinus, lo que se demostró por prueba cutánea (PC) y RAST (radio allergo sorbent test). Los controles fueron negativos a estos alergenos por PC y RAST.

Los niveles totales de IgE (IgE PRIST- Pharmacia) en los pacientes estuvieron por encima de $800 \mathrm{KU} /$ $L y$, en los controles, los valores estuvieron entre 80 y $300 \mathrm{KU} / \mathrm{L}$, rango ya establecido por nuestro laboratorio para la población sana. EI RAST para $\lg$ E específica para $D$. pteronnyssinus se hizo por ensayo inmunoenzimático (Phadezim-RASTPharmacia). EI RAST para B. tropicalis se hizo usando platos de microtitulación y un anticuerpo anti-lgE marcado con $\mathrm{I}^{123}$, como se describió previamente (15). Adicionalmente, se usó un extracto de ácaro total para evaluar la respuesta de lgE específica contra un alergeno recombinante del ácaro B. tropicalis llamado BtM. Este alergeno fue obtenido de una biblioteca de CDNA de $B$. tropicalis (16). La unión de la IgE a BtM fue detectada por inmunoensayo en placa. En el grupo de pacientes, un subgrupo de 40 (42\%) tenían anticuerpos 
IgE contra BtM. Solamente 62 sueros del grupo control, pudieron ser probados para este alergeno. Entre estos, 4 fueron positivos, siendo finalmente los verdaderos controles un subgrupo de 58 individuos con respuesta negativa a BtM.

Tipificación molecular de los genes HLA y TAP: la tipificación molecular de los genesTAP se hizo por PCR /SSO como fue descrita previamente (17). Los primers para la PCR fueron seleccionados con el fin de amplificar fragmentos que no excedieran los $400 \mathrm{pb}$ y que, además,cubrieran todas las regiones polimórficas ubicadas en la región trasmembrana y citoplasmática de los genes TAP. EI ADN amplificado fue desnaturalizado con $\mathrm{NaOH} 0,45 \mathrm{~N}$ e hibridizado con sondas de oligonucleótidos marcadas radioactivamente en el extremo 5'. Cuatro sondas que cubrían las posiciones 333 y 637 permitieron la caracterización de los alelos TAP1. Seis sondas que cubrían las posiciones polimórficas 379,665 y 687 , los de TAP2. Las señales de hibridización se detectaron exponiendo las membranas con películas Kodak O-MAT, entre 2 y 16 horas. La tipificación HLA clase II también se hizo mediante PCR/SSO siguiendo el protocolo del XITaller Internacional de Histocompatibilidad (18).

Tipificación molecular LMP2: el polimorfismo dialélico del codón 60 (Arg-His) en el gen LMP2 fue detectado por PCR/RFLP como se describió previamente (19). La reacción de PCR fue hecha en 30 ciclos así: un ciclo de tres temperaturas $\left(94^{\circ} \mathrm{C}\right.$ for 5 minutos, $55^{\circ} \mathrm{C}$ por 90 segundos, $72^{\circ} \mathrm{C}$ por 2 minutos) y 29 ciclos de tres temperaturas $\left(94^{\circ} \mathrm{C}\right.$ por 90 segundos, $55^{\circ} \mathrm{C}$ por 90 segundos y $72^{\circ} \mathrm{C}$ por 2 minutos). El producto amplificado fue digerido con 4 unidades de Hhal por 2 horas a $37^{\circ} \mathrm{C}$. El alelo Arg-60, designado como LMP-R, contiene el sitio para la enzima Hhal en el codón 60 , mientras que LMP2-His-60, designado como LMP2-H no. La presencia de los fragmentos de $1167 \mathrm{pb}$ y $1088 \mathrm{pb}$ después de la digestión con Hhal representan al alelo LMP2-R. Cuando se obtiene un fragmento de $2255 \mathrm{pb}$, este corresponde al alelo LMP2-H (figura 1).

Análisis estadístico: las frecuencias de los genes HLA clase II, TAP y LMP2 fueron obtenidas por conteo directo. La comparación de las frecuen-

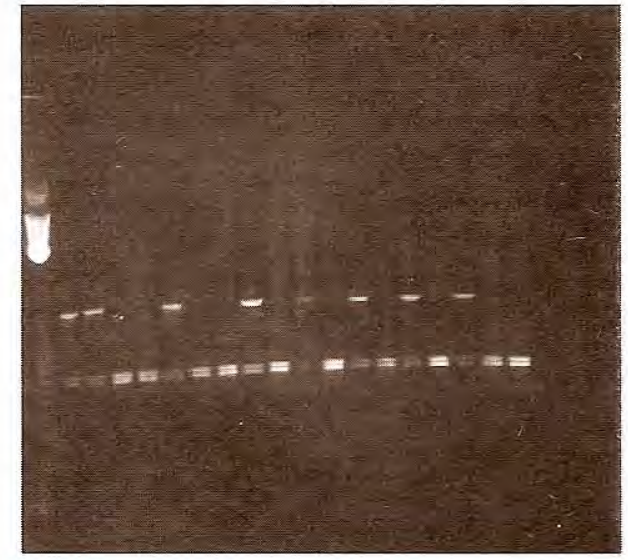

Figura 1. Electroforesis en gel de agarosa de la tipificación del gen LMP2 por PCR/RFLP donde se observan los diferentes genotipos encontrados. Carril 1: marcador de peso molecular; los carriles donde aparecen 3 bandas corresponden al genotipo LMP2*R/H y en los que se observan 2 bandas corresponden a LMP2*R/R.

cias alélicas entre pacientes y controles fue obtenida por análisis de chi cuadrado, corrigiendo la $p$ al multiplicar su valor por el número de alelos estudiados $(\mathrm{Pc})$. El desequilibrio de ligamiento entre dos loci genéticos y los valores de delta se obtuvieron por el metodo de Mattiuz (20).

\section{Resultados y discusión}

La distribución de los alelos TAP1 y TAP2 en pacientes y controles aparece en el cuadro 1 . Se encontraron cuatro alelos TAP1, confirmando los datos publicados previamente $(17,21,22)$. Se observaron tres alelos TAP2. Adicionalmente, en 6 pacientes y 2 controles fue identificado el alelo TAP2H (figura 2). No se encontró diferencia estadísticamente significativa en la distribución de los alelos TAP1 y TAP2 entre los pacientes que presentaban hiperrespuesta de lgE al extracto de ácaros y los controles. Sin embargo, la distribución de valina en la posción 333 del TAP1 fue significativamente más frecuente en controles que en pacientes asmáticos $(\mathrm{Pc}=0,03)$. No hubo asociación significativa cuando se compararon las frecuencias de los alelos TAP entre los subgrupos de pacientes reactivos al recombinante BtM y los controles no reactivos.

Nosotros habíamos descrito previamente una asociación entre el gen HLA-DRB $1{ }^{*} 03$ y la respuesta 
Cuadro 1. Distribución de los alelos TAP entre pacientes con asma alérgica y controles.

\begin{tabular}{|c|c|c|c|}
\hline cor & $\begin{array}{l}\text { Pacientes } \\
\text { asma alérgica }\end{array}$ & Controles & $\mathrm{Pc}$ \\
\hline Alelos TAP1 & $n=192$ & $n=194$ & \\
\hline${ }^{*} 0101$ & $52,6 \%$ & $56,2 \%$ & $\mathrm{~ns}$ \\
\hline${ }^{*} 02011$ & $35,9 \%$ & $34,9 \%$ & $\mathrm{~ns}$ \\
\hline${ }^{*} 0301$ & $5,2 \%$ & $3,8 \%$ & ns \\
\hline${ }^{*} 0401$ & $6,3 \%$ & $4,7 \%$ & ns \\
\hline Alelos TAP2 & $n=142$ & $n=130$ & \\
\hline A & $31,5 \%$ & $30,5 \%$ & ns \\
\hline B & $44,3 \%$ & $46,6 \%$ & $\mathrm{~ns}$ \\
\hline C & $16,5 \%$ & $18,0 \%$ & ns \\
\hline$H$ & $7,7 \%$ & $4,9 \%$ & $\mathrm{~ns}$ \\
\hline
\end{tabular}

de $\lg E$ contra alergenos de ácaros $(11,12)$; por tanto, se analizó la relación entre los alelos TAP y HLA clase II (DRB1 y DQB1). No se encontró desequilibrio de ligamiento entre $\mathrm{DRB} 1^{*} 03$ o cualquier otro alelo DRB1 y los genes TAP. Además, en nuestro principal grupo control hallamos algunos haplotipos DRB1-TAP, TAP1-TAP2, DQB1-TAP descritos en otras poblaciones $(21,22)$.

Por otro lado, hallamos desequilibrio de ligamiento entre los genes HLA DQB1 y algunos alelos TAP ya publicados (cuadro 2). Es interesante que el haplotipo más sobresaliente de la población caucásica (23), TAP2A-DR3-DQ2, no fue observado en este estudio.

La distribución de los alelos del gen LMP2 se muestra en el cuadro 3. No hubo diferencia esta-

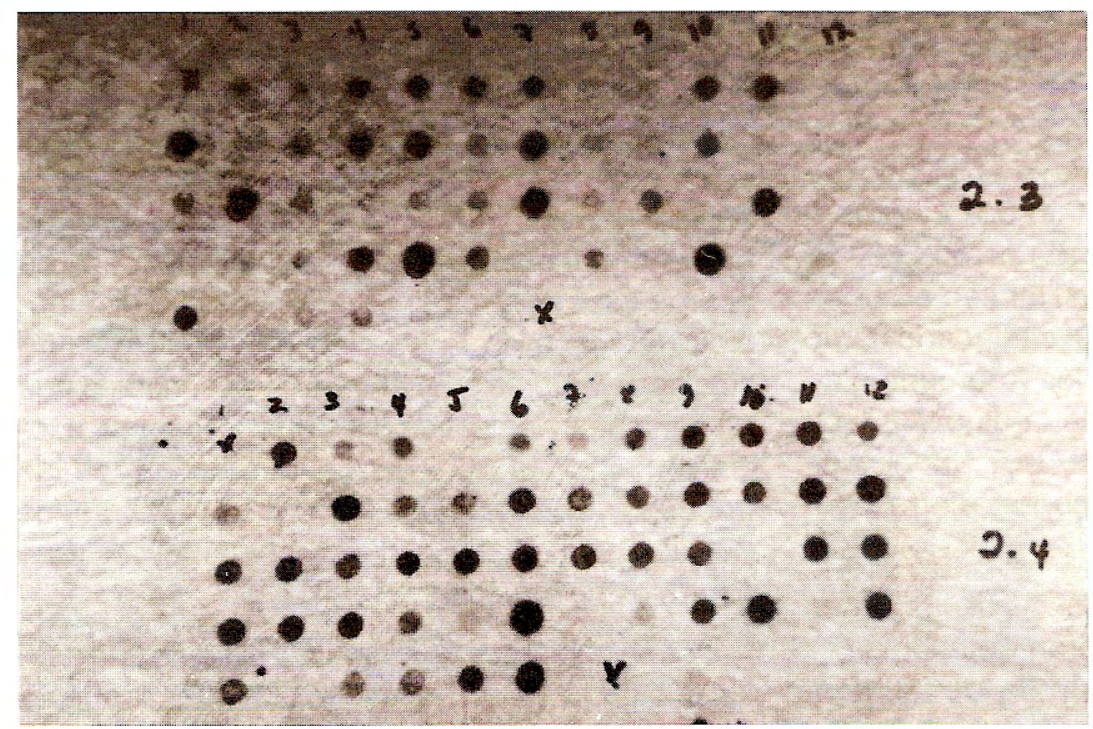

Cuadro 2. Haplotipos DR-DQ-TAP.

\begin{tabular}{rrr}
\hline DRB1 & TAP1 & Chi $^{2}$ \\
${ }^{*} 1101$ & ${ }^{*} 0101$ & $\mathrm{~ns}$ \\
${ }^{*} 1501$ & ${ }^{*} 0301$ & $\mathrm{~ns}$ \\
${ }^{*} 1601$ & ${ }^{*} 0301$ & $\mathrm{~ns}$ \\
${ }^{*} 4$ & ${ }^{*} 0301$ & $\mathrm{~ns}$ \\
${ }^{*} 8$ & ${ }^{*} 02011$ & $\mathrm{~ns}$ \\
${ }^{*} 1001$ & ${ }^{*} 0101$ & $\mathrm{~ns}$ \\
${ }^{*} 1406$ & ${ }^{*} 0301$ & $\mathrm{~ns}$ \\
${ }^{*} 0101$ & ${ }^{*} 02011$ & $\mathrm{~ns}$ \\
& & \\
DQB1 & TAP1 & Chi \\
${ }^{*} 0302$ & ${ }^{*} 02011$ & 5,51 \\
${ }^{*} 0402$ & ${ }^{*} 0101$ & 4,07 \\
${ }^{*} 0602$ & ${ }^{*} 02011$ & 7,44 \\
& & \\
DQB1 & TAP2 & Chi \\
${ }^{*} 0303$ & B & 5,2 \\
${ }^{*} 1001$ & A & 3,8 \\
\hline
\end{tabular}

dísticamente significativa en la distribución de estos alelos entre pacientes y controles. Además, tampoco hubo diferencia significativa en la distribución de los alelos LMP2 al comparar los pacientes HLA DRB $1^{\star} 03$ positivos o negativos, con respecto a los controles.

Ya que los genes TAP y LMP están ubicados en la región HLA clase II, entre los genes HLA DR y $D Q, y$ sus productos participan en el procesamiento antigénico, son buenos candidatos para ser genes asociados a enfermedades, lo cual ha sido sugerido por algunos estudios. Aunque el
Figura 2. Tipificación del gen TAP2 con dos sondas denominadas 2.3 y 2.4. Se observan las señales de hibridización de 50 pacientes con asma alérgica. Los patrones de reacción observados con éstas y las restantes 4 sondas permitieron definir los alelos. 
Cuadro 3. Distribución de los genes LMP2 en pacientes con asma alérgica y controles.

\begin{tabular}{lccc}
\hline $\begin{array}{l}\text { LMP2 } \\
\text { genotipo }\end{array}$ & $\begin{array}{c}\text { Pacientes } \\
(n=75)\end{array}$ & $\begin{array}{c}\text { Controles } \\
(n=87)\end{array}$ & pc \\
\hline LMP2*H/H & $5(6,7 \%)$ & $9(10,4 \%)$ & $n s$ \\
LMP2*R/R & $32(42,7 \%)$ & $39(44,8 \%)$ & $n s$ \\
LMP2 $^{*} R / H$ & $38(50,6 \%)$ & $39(44,8 \%)$ & $n s$ \\
\hline
\end{tabular}

procesamiento y presentación de los alergenos sigue la vía endosómica, utilizando las moléculas HLA clase II, los productos de los genes TAP pueden participar en el transporte de los péptidos exógenos, en un proceso dependiente de las moléculas HLA clase I (24). Además, algunos péptidos endógenos pueden ser presentados por moléculas HLA clase II, dependiendo de la proteína transportadora $\operatorname{TAP}(25,26)$.

Sin embargo, nuestros resultados no muestran asociación alguna entre la respuesta IgE contra alergenos de ácaros y los alelos TAP y LMP2. Además, no hallamos ningún desequilibrio de ligamiento entre estos genes y los clase II descritos previamente como asociados con la respuesta de IgE contra alergenos de ácaros. Lo anterior sustenta la hipótesis de que los genes HLA clase II son los que definen la asociación primaria descrita para esta respuesta. En este sentido, definir la influencia que tiene el polimorfismo de otros locigenéticos relacionados (27) sobre la respuesta de IgE contra alergenos podría aumentar nuestro conocimiento acerca del papel real de la región HLA en la genética del asma alérgica.

En conclusión, este estudio describe la distribución de los alelos TAP y LMP2 en la población mulata normal y en pacientes con asma alérgica. Igualmente, presentamos la distribución de haplotipos entre los alelos de estos genes con alelos HLA DRB1 y DQB1. No se encontró asociación entre los genes TAP y LMP2 y la respuesta inmunológica lgE contra alergenos de ácaros.

\section{Agradecimientos}

Esta investigación fue financiada por la Fundación para la Promoción de la Investigación y la Tecnología del Banco de la República, Colombia, y la Universidad de Cartagena.

\section{Referencias}

1. Bodmer JG, Marsh SG, et al. Nomenclature for factors of the HLA system, 1991. Tissue Antigens 1992;39:16173.

2. Monaco J, Cho S, Attaya M. Transport protein genes in the murine MHC: possible implications for antigen processing. Science 1990;250:1723-6.

3. Trowsdale J, Hanson I, Mockridge I, Townsend A, Kelly $\mathbf{A}$. Sequences encoded in the class II region of the MHC related to the "ABC" superfamily of transporters. Nature 1990;348:741-4.

4. Powis S, Mockridge I, Kelly A, Kerr L, Glynne R, Gileadi U, Beck S, Trowsdale J. Polymorphism in a second $A B C$ transporter gene located within the class Il region of the human major histocompatibility complex. Proc Nat Acad Sci (USA) 1990;89:1463-7.

5. Jackson D, Capra D. TAP1 alleles in insulin-dependent diabetes mellitus: a newly defined centromeric boundary of disease susceptibility. Proc Nat Acad Sci (USA) 1993;90:11079-83.

6. Fernandez-Viña MA, Fink CW, Sang S, Stastny P. Peptide transporter genes in susceptibility to juvenile arthritis. Hum Immunol 1993,Supl.1;37:58.

7. Barron KS, Carrington M, Reveile JD, Robinson MA. Polymorphism in TAP genes in Reiter's sindrome. Hum Immunol 1993, supl. 1;37:107.

8. Kellar Wood HF, Powis SH, Gray J, Compston DAS. MHC-enconded TAP1 and TAP2 dimorphisms in multiple sclerosis. Tissue Antigens 1994;43:129-32.

9. Caraballo L, Marrugo J, Erlich H, Pastorizo M. HLA antigens in the population of Cartagena (Colombia). Tissue Antigens 1992;39:128-33.

10. Caraballo L, Marrugo J, Jiménez S, Angelini G, Ferrara GB. Frequency of DPB $1^{*} 0401$ is significantly decreased in patients with allergic asthma in mulatto population. Hum Immunol 1991;32:157-61.

11. Caraballo L, Puerta L, Jiménez S, Martínez B, Moreno L. An immunogenetic study of the $\operatorname{lgE}$ resposiveness to allergens of Blomia tropicalis (Bt) in patients with allergic asthma. J Allergy Clin Immuno 1993; [Abstract]91:335.

12. Caraballo L, Martínez B, Jiménez S, Madrigal A, Forde $\mathrm{H}$. Immunogenetics and immune response to mite allergens. In: Sastre J, Basomba A, eds. Proceedings I, XVI European Congress of Allergology and Clinical Immunology-EACl'95. Bologna: Monduzzi Editore S.P.A.; 1995:265-71.

13. Van-Endert P, López M, Patel S, Monaco J, McDevitt H. Genomic polymorphism, recombination and linkage disequilibrium in human major histocompatibility complex- 
encoded antigen-processing genes. Proc Natl Acad Sci (USA) 1992;82:11594-7.

14. Busse WW, Reed CE. Asthma: definition and pathogenesis. In: Middleton E, Reed CE, Ellis EF, Adkinson NF, Yunginger JW, eds. Allergy principles and practice. St. Louis: Mosby; 1988.

15. Puerta L, Fernandez-Caldas E, Caraballo L, Lockey R. Sensitization to Blomia tropicalis and Lepydoglyphus destructor in Dermatophagoides $\mathrm{spp}$. allergic individuals. J Allergy Clin Immunol 1991;88:943-50.

16. Caraballo L, Avjioglu A, Marrugo J, Puerta L, Marsh D. Cloning and expression of complementary DNA coding for an allergen with common antibody binding specificities with three allergens of the house dust mite Blomia tropicalis. J Allergy Clin Immunol 1996;98:573-9.

17. Colonna M, Breshnaham M, Bahram S, Strominger J. Allelic variants of the human putative peptide transporters involved in antigen processing. Proc Nat Acad Sci (USA) 1992;89:3932-6.

18. Tsuji K, Aizawa M, Sasazuki T. Proceedings of the eleventh International Histocompatibility Workshop and Conference. Oxford University Press Eds. Japan; 1992.

19. Buney R, Pile K, Gibson K, Calin A, Kennedy L, Sinnott P, Powis S, Wordsworth B. Analysis of the $\mathrm{MHC}$ encoded component of the class I antigen processing pathway in ankylosing spondylitis. Ann Rheum Dis 1994;53(1):5860.

20. Mattiuz P, Ihde D, Piazza A, Cepellini R, Bodmer W. New approaches to the population genetics and segregation analysis of the HLA system. In: Kissmeyer-Nielsen F, ed. Histocompatibility testing 1970. Copenhagen: Munksgaard;1970:193-206.

21. Ploski R, Undlien D, Vinje O, Forre O, Thorsby E, Ronningen $\mathbf{K}$. Polimorphism of human major histocompatibility complex-encoded transporter associated with antigen processing (TAP) genes and susceptibility to juvenil rheumatoid arthritis. Human Immunol 1994;39:54-60.

22. Powis S, Tonks S, Mockridge I, Kelly A, Bodmer $\mathrm{J}$, Trowsdale J. Alleles and haplotypes of the MHCencoded ABC transporters TAP1 and TAP2. Immunogenetics 1993;37:373-80.

23. Powis S, Cooper M, Trowsdale J, Zhu Z, Valanakis J. Major histocompatibility haplotypes associated with Immunoglobulin-A-deficiency: analysis of the peptide transporter genes TAP1 and TAP2. Tissue Antigens 1994;43:261-5.

24. Gonzalez Escribano M, Yélamos J, García-Loza no J., et al. Letter to the Editor. Tissue Antigens 1995;45:77-8.

25. Malnati MS, Morti M, La Vante T, et al. Processing pathways for presentation of cytosolic antigen to $\mathrm{MHC}$ class II restricted T cell. Nature 1992;357:702-704.

26. Kovacsovics-Bankowski M, Rock K. A phagosometo-cytosol pathway for exogenous antigens presented on MHC Class I molecules. Science 1995;267:243-45.

27. Fling S, Arp B, Plous D. HLA-DMA and DMB are both required for $\mathrm{MHC}$ class II/peptide complex formation in antigen-presenting cells. Nature 1994;368:554-8. 